\title{
Mosquito vector proteins homologous to a1-3 galactosyl transferases of tick vectors in the context of protective immunity against malaria and hypersensitivity to vector bites
}

Ranjan Ramasamy* ${ }^{*}$

\begin{abstract}
Background: An epitope, Gala1-3Galß1-4GlcNAc-R, termed a-gal, is present in glycoconjugates of New World monkeys (platyrrhines) and other mammals but not in hominoids and Old World monkeys (catarrhines). The difference is due to the inactivation of a1-3 galactosyl transferase (a1-3 GT) genes in catarrhines. Natural antibodies to a-gal are therefore developed in catarrhines but not platyrrhines and other mammals. Hypersensitivity reactions are commonly elicited by mosquito and tick vector bites. IgE antibodies against a-gal cause food allergy to red meat in persons who have been exposed to tick bites. Three enzymes synthesising the terminal a1-3-linked galactose in a-gal, that are homologous to mammalian a and B1-4 GTs but not mammalian a1-3 GTs, were recently identified in the tick vector Ixodes scapularis. IgG and IgM antibodies to a-gal are reported to protect against malaria because mosquito-derived sporozoites of malaria parasites express a-gal on their surface. This article explores the possibility that the a-gal in sporozoites are acquired from glycoconjugates synthesised by mosquitoes rather than through de novo synthesis by sporozoites.

Methods: The presence of proteins homologous to the three identified tick a1-3 GTs and mammalian a1-3 GTs in two important mosquito vectors, Aedes aegypti and Anopheles gambiae, as well as Plasmodium malaria parasites, was investigated by BLASTp analysis to help clarify the source of the a-gal on sporozoite surfaces.

Results: Anopheles gambiae and Ae. aegypti possessed several different proteins homologous to the three I. scapularis proteins with a1-3 GT activity, but not mammalian a1-3 GTs. The putative mosquito a1-3 GTs possessed conserved protein domains characteristic of glycosyl transferases. However, the genus Plasmodium lacked proteins homologous to the three I. scapularis proteins with a1-3 GT activity and mammalian a1-3 GTs.

Conclusions: The putative a1-3 GTs identified in the two mosquito vectors may synthesise glycoconjugates containing a-gal that can be transferred to sporozoite surfaces before they are inoculated into skin during blood feeding. The findings merit further investigation because of their implications for immunity against malaria, hypersensitivity to mosquito bites, primate evolution, and proposals for immunisation against a-gal.
\end{abstract}

*Correspondence: ranjanramasamy@idfishtechnology.com

ID-FISH Technology, 556 Gibraltar Drive, Milpitas CA95035, USA

(c) The Author(s) 2021. This article is licensed under a Creative Commons Attribution 4.0 International License, which permits use, sharing, adaptation, distribution and reproduction in any medium or format, as long as you give appropriate credit to the original author(s) and the source, provide a link to the Creative Commons licence, and indicate if changes were made. The images or other third party material in this article are included in the article's Creative Commons licence, unless indicated otherwise in a credit line to the material. If material is not included in the article's Creative Commons licence and your intended use is not permitted by statutory regulation or exceeds the permitted use, you will need to obtain permission directly from the copyright holder. To view a copy of this licence, visit http://creativeco mmons.org/licenses/by/4.0/. The Creative Commons Public Domain Dedication waiver (http://creativecommons.org/publicdomain/ zero/1.0/) applies to the data made available in this article, unless otherwise stated in a credit line to the data. 
Keywords: a-Gal, a-Gal syndrome, a1-3 Galactosyl epitopes, Antibodies to a-gal, Galactosyl transferases, Hypersensitivity, Immune protection, Malaria, Mosquito vectors, Tick vectors

Infected female vector mosquitoes taking a human blood meal transmit parasites (e.g. Plasmodium malaria parasites) and arboviruses (e.g. dengue virus) that cause serious diseases of global concern. Mosquitoes inject saliva into the skin to facilitate blood feeding. Components of mosquito saliva, that include many proteins, can cause immediate and delayed hypersensitivity reactions involving IgE and IgG antibodies as well as T lymphocytes in the skin at the bite site, resulting sometimes in severe delocalised and systemic pathology [1]. Ticks are also arthropod vectors that transmit parasites (e.g. Babesia species), bacteria (e.g. Borrelia species), and viruses (e.g. tick-borne encephalitis virus) acquired from wild animals to cause globally prevalent human diseases [2]. Ticks are rare opportunistic feeders on humans, and therefore, less is known about hypersensitivity reactions to tick salivary components injected into humans during blood feeding. It is, however, known that immunisation of guinea pigs with tick saliva glycoproteins produces immune-mediated rejection of ticks feeding on guinea pigs [3].

A type of food allergy to mammalian meat is now recognised as a type 1 hypersensitivity reaction that occurs in a proportion of people who have been exposed to tick bites [4]. IgE antibodies to the trisaccharide epitope Gal $\alpha 1-3 G a l \beta 1-4 G l c N A c-R$ ( $\alpha$-gal), initially elicited by molecules in tick saliva containing $\alpha$-gal, bind to $\alpha$-gal from red meat and cause the hypersensitivity, which has been termed the $\alpha$-gal syndrome (AGS) [4]. AGS can produce clinical manifestations ranging from urticaria to fatal anaphylaxis [4]. Human serum, however, contains natural antibodies of IgG, IgM and IgA isotypes to $\alpha$-gal that constitute approximately $1 \%$ of all immunoglobulins in serum, and these antibodies are elicited by $\alpha$-gal-containing glycolipids present in commensal gut bacteria [5-8]. Humans, apes and Old World monkeys (termed catarrhines) lack $\alpha$-gal and are therefore able to the produce antibodies to $\alpha$-gal, while new world monkeys (termed platyrrhines), lemurs and other mammals possess $\alpha$-gal and, because of self-tolerance, are unable to produce antibodies to $\alpha$-gal [5-8]. Inactivating mutations in $\alpha 1-3$ galactosyl transferases ( $\alpha 1-3 \mathrm{GTs}$ ), estimated to have occurred 20-28 million years ago as two separate events in Old World monkeys and hominids during catarrhine evolution, are responsible for this difference [9].

The $\alpha$-gal in the tick vector Ixodes scapularis has been shown to be synthesised by two tick enzymes that are homologous to an $\alpha 1-4$ GT in humans responsible for synthesising globosides (also termed Gb3 synthase), and a third enzyme which is homologous to a human $\beta 1-4$ GT [10]. The $\alpha 1-3$ GT activity of the three I. scapularis enzymes has been attributed to an altered specificity that arose during evolution [10], which may have been facilitated by an expansion of the numbers of $\alpha$ and $\beta$ GTs in I. scapularis [10]. The presence of $\alpha$-gal in the salivary glands of Anopheles mosquito vectors and in Plasmodium sporozoites obtained from Anopheles salivary glands has been reported, but whether the $\alpha$-gal found on sporozoites was synthesised by sporozoites or acquired from the Anopheles vector was not established [11]. Because of important implications for protective immunity against mosquito-borne pathogens, hypersensitivity due to mosquito bites, and primate evolution, the possible presence of $\alpha 1-3$ GTs in the principal African malaria vector Anopheles gambiae, the primary global arboviral vector Aedes aegypti and the genus Plasmodium was investigated by in silico protein sequence homology analysis.

The existence of proteins with homology to the three identified tick $\alpha 1-3$ GTs [10] and three murine $\alpha 1-3$ GTs was explored by NCBI BLASTp searches (https://blast. ncbi.nlm.nih.gov/Blast.cgi?PAGE=Proteins) against all non-redundant protein sequences of An. gambiae (NCBI taxid 7165), Ae. aegypti (NCBI taxid 7159) and the genus Plasmodium (NCBI taxid 5820). These protein sequences available at NCBI are mostly predicted from gene sequences obtained by whole genome sequencing. Search parameters utilised in the BLASTp analysis were as follows: matrix-BLOSUM62; gap costs-existence 11, extension 1; alignment initiation length 6; conditional compositional score matrix adjustment; threshold $E$ value 0.05 . The three $I$. scapularis protein sequences used as queries in BLASTp were obtained from the UniProtKB/ Swiss-Prot database (https://www.uniprot.org) using the VectorBase I. scapularis gene identities from reference 10. Two of the three I. scapularis enzymes with $\alpha 1-3$ GT activity are annotated in the UniProtKB/Swiss-Prot protein database as $\alpha 1-4 \mathrm{~N}$-acetylglucosaminyl transferases (B7QKR3 and BFPLD1) and the third as a xylosylprotein $\beta$ galactosyl transferase (B7PFJ6).

Protein sequences from the three known functional mammalian $\alpha 1-3$ GTs from Mus musculus were also used in BLASTp analysis against non-redundant protein sequences of An. gambiae (NCBI taxid 7165), Ae. aegypti (NCBI taxid 7159) and the genus Plasmodium (NCBI taxid 5820) using the BLASTp parameters described above. The murine $\alpha 1-3$ GT protein sequences used were 
as follows: (i) glycoprotein $\alpha 1-3$ galactosyl transferase with NCBI reference NP_001139293.1; (ii) cis AB transferase with NCBI reference BAB20560.1; (iii) isoglobotriasoylceramide synthase from UniProtKB/Swiss-Prot with the reference Q3VIN9. Additional IDs for mosquito proteins identified through BLASTp were obtained from VectorBase (https://vectorbase.org/vectorbase/app).

Conserved functional domains in homologous proteins and phylogenetic trees for them in the NCBI Tree viewer using the nearest-neighbour-joining tree method and Kimura distance option were determined through the BLASTp output search options.

Proteins homologous to the three I. scapularis enzymes were not detected in the genus Plasmodium by BLASTp analysis at $E \leq 0.05$. Additionally, BLASTp analysis of the genus Plasmodium, An. gambiae and Ae. aegypti with the three functional mammalian $\alpha 1-3$ GTs did not identify homologous proteins at $E \leq 0.05$. However, both $A n$. gambiae and Ae. aegypti possessed several unique proteins that were homologous to each of the three $I$. scapularis enzymes with $\alpha 1-3$ GT activity, with $E$ values that were much less than 0.05 , showing that all observed sequence homologies were highly significant (Table 1). The high alignment scores and query cover showed that the homologies between the tick and mosquito proteins extended over most of the length of each pair of proteins that were compared. Furthermore, no other unique homologous Aedes or Anopheles proteins other than those listed in Table 1 were identified by BLASTp analysis against the three $I$. scapularis GTs. The two enzymes with $\alpha 1-3$ GT activity but annotated as $\alpha 1-4 \mathrm{~N}$-acetylglucosaminyl transferases from I. scapularis identified a set of three Ae. aegypti proteins, with each of the three proteins having different $E$ values and percent identities against the two I. scapularis enzymes. These two I. scapularis enzymes also identified a set of four An. gambiae proteins with each of the four proteins having different $\mathrm{E}$ values and percent identities against the two I. scapularis

Table 1 Aedes aegypti and Anopheles gambiae homologues of Ixodes scapularis enzymes with a1-3 galactosyl transferase activity identified by NCBI BLASTp analyses

\begin{tabular}{|c|c|c|c|c|c|c|}
\hline NCBI accession ID (VectorBase protein ID) & Evalue & Percent identity & $\begin{array}{l}\text { Query cover } \\
(\%)\end{array}$ & Max. score & Total score & $\begin{array}{l}\text { Accession } \\
\text { length }\end{array}$ \\
\hline \multicolumn{7}{|c|}{ Ae. aegypti homologues of I. scapularis B7QKR3 (ISCW024908) annotated as a1-4 N-acetylglucosaminyl transferase (length 300 amino acids) } \\
\hline XP_001654206.1* (AAEL001900-PA) & $3.00 E-25$ & 28.47 & 90 & 103 & 103 & 371 \\
\hline XP_001650194.1 (AAEL005019-PA) & $1.00 \mathrm{E}-18$ & 24.18 & 88 & 85.5 & 85.5 & 354 \\
\hline XP_001654207.2 (AAEL001895-PA) & $2.00 E-18$ & 23.55 & 88 & 85.1 & 85.1 & 404 \\
\hline \multicolumn{7}{|c|}{ Ae. aegypti homologues of I. scapularis B7PLD1 (ISCW006262) annotated as a1-4 N-acetylglucosaminyl transferase (length 344 amino acids) } \\
\hline XP_001650194.1 (AAEL005019-PA) & $2.00 E-41$ & 32.86 & 79 & 148 & 148 & 354 \\
\hline XP_001654206.1* (AAEL001900-PA) & $6.00 E-34$ & 27.11 & 78 & 129 & 129 & 371 \\
\hline XP_001654207.2 (AAEL001895-PA) & $1.00 E-24$ & 25.75 & 80 & 103 & 103 & 404 \\
\hline \multicolumn{7}{|c|}{ Ae. aegypti homologues of I. scapularis B7PFJ6 (ISCW003979) annotated as xylosylprotein $\beta$ galactosyl transferase (length 290 amino acids) } \\
\hline XP_001653000.2* (AAEL007895-PA) & $6.00 E-102$ & 48.79 & 98 & 301 & 301 & 313 \\
\hline XP_021707280.1 (AAEL019794-PA) & $8.00 E-32$ & 40.91 & 52 & 123 & 123 & 485 \\
\hline XP_021703090.1 (AAEL021029) & $2.00 E-29$ & 37.91 & 51 & 114 & 114 & 318 \\
\hline \multicolumn{7}{|c|}{ An. gambiae homologues of I. scapularis B7QKR3 (ISCW024908) annotated as a1-4 N-acetylglucosaminyl transferase (length 300 amino acids) } \\
\hline XP_555205.1* (AGAP008260-PA) & $8.00 E-23$ & 25.46 & 88 & 96.7 & 96.7 & 404 \\
\hline XP_317211.3 (AGAP008258-PA) & $3.00 E-22$ & 25.27 & 88 & 94.7 & 94.7 & 358 \\
\hline XP_001237894.2 (AGAP008259-PA) & $5.00 E-22$ & 26.74 & 88 & 93.2 & 93.2 & 302 \\
\hline XP_555204.2 (AGAP008261-PA) & $1.00 \mathrm{E}-07$ & 22.74 & 90 & 52 & 52 & 347 \\
\hline \multicolumn{7}{|c|}{ An. gambiae homologues of I. scapularis B7PLD1 (ISCW006262) annotated as a1-4 N-acetylglucosaminyl transferase (length 344 amino acids) } \\
\hline XP_001237894.2 (AGAP008259-PA) & $4.00 \mathrm{E}-49$ & 33.22 & 83 & 166 & 166 & 302 \\
\hline XP_317211.3 (AGAP008258-PA) & $3.00 E-44$ & 31.79 & 83 & 155 & 155 & 358 \\
\hline XP_555205.1* (AGAP008260-PA) & $2.00 E-41$ & 31.58 & 84 & 149 & 149 & 404 \\
\hline XP_555204.2 (AGAP008261-PA) & $5.00 \mathrm{E}-17$ & 27.46 & 78 & 80.1 & 80.1 & 347 \\
\hline \multicolumn{7}{|c|}{ An. gambiae homologues of I. scapularis B7PFJ6 (ISCW003979) annotated as xylosylprotein $\beta$ galactosyl transferase (length 290 amino acids) } \\
\hline XP_318033.4 (AGAP0048781-PA) & $1.00 \mathrm{E}-30$ & 31.28 & 72 & 116 & 116 & 308 \\
\hline XP_001689009.1 (AGAP008285-PA) & $1.00 \mathrm{E}-27$ & 32.86 & 71 & 108 & 108 & 322 \\
\hline
\end{tabular}

Data are the results of BLASTp searches for different mosquito proteins with significant homology $(E \leq 0.05)$ to the three Ixodes scapularis enzymes. Asterisks indicate proteins that were also identified in reference 10. The UniProtKB/Swiss-Prot IDs with their VectorBase gene IDs in parentheses are shown for I. scapularis proteins. Homologous mosquito proteins are shown with their NCBI accession IDs with VectorBase IDs in parentheses 
enzymes. This suggests that the three putative Ae. aegypti GTs identified in this way have close sequence homology with each other as do the four putative An. gambiae GTs with each other, and also that the two I. scapularis

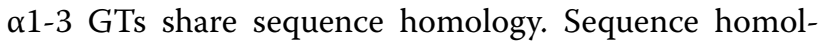
ogy also exists within the three putative Ae. aegypti GTs and within the two putative An. gambiae GTs that were identified using the single I. scapularis protein annotated as a xylosylprotein $\beta$ galactosyl transferase but possessing $\alpha 1-3$ GT activity. These homologies are further illustrated in the phylogenetic trees constructed for each of the three I. scapularis enzymes and their homologous Ae. aegypti and An. gambiae proteins (Additional file 1).

The two $I$. scapularis enzymes annotated as $\alpha 1-4$ $N$-acetylglucosaminyl transferases but possessing $\alpha 1-3$ GT activity, and all their homologous putative Ae. aegypti and An. gambiae GTs shown in Table 1 possessed two conserved protein domains: (i) pfam 04572 characteristic of globoside synthases; and (ii) cl 19952 which contains a UDP-galactose binding region with a DXD amino acid motif. The I. scapularis protein annotated as a xylosylprotein $\beta$ galactosyl transferase but with $\alpha 1-3$ GT activity and all the identified homologous putative Ae. aegypti and An. gambiae a1-3 GTs shared the conserved protein domain cl 11394 which is representative of glycosyl transferase family A with a GT-A type of structural fold, as well as the DXD amino acid motif which participates in binding divalent metal ions essential for GT activity [12].

Based on the binding of B4 lectin from Bandeiraea (Griffonia) simplicifolia which specifically recognises terminal $\alpha$-galactosyl residues and a monoclonal antibody specific for the $\alpha$-gal epitope, as well as abrogation of their binding by prior $\alpha$-galactosidase treatment, $\alpha$-gal was reported to be present in the salivary glands of Anopheles vectors as well as Plasmodium falciparum and Plasmodium berghei sporozoites obtained from Anopheles salivary glands [11]. Investigations of the protection conferred by antibodies to $\alpha$-gal against infection with the murine malaria parasite $P$. berghei in genetically engineered mice unable to synthesise $\alpha$-gal were then undertaken [11]. Antibodies to $\alpha$-gal in such mice conferred partial protection against blood-stage infection by $P$. berghei sporozoites inoculated by mosquito bites, but not intravenously injected $P$. berghei sporozoites [11]. The study produced evidence supporting roles for IgM and some IgG isotypes of antibodies to $\alpha$-gal, complement and phagocytic cells in immune damage to $P$. berghei sporozoites at the skin inoculation site [11]. However, it was not able to definitively differentiate between an origin for the $\alpha$-gal-containing glycoconjugates in anopheline salivary glands that become bound to sporozoite surfaces, and the de novo synthesis of $\alpha$-gal by sporozoites [11]. Plasmodium berghei sporozoites reside in the skin for a considerable length of time after being inoculated by a biting anopheline, and this can facilitate immunological damage initiated by antibodies to $\alpha$-gal $[13,14]$.

Early studies had suggested the presence of $\alpha$-gal in cultured asexual blood stages of the human malaria parasite $P$. falciparum based on sensitivity to $\alpha$-galactosidase treatment $[15,16]$, binding of Bandeiraea simplicifolia lectin isoform B4 [16, 17], and weak complementdependent inhibition of asexual blood stage growth by natural antibodies to $\alpha$-gal [7]. However, subsequent experiments showed that these were probably due to non-specific binding of the Bandeiraea simplicifolia lectin isoform B4 to $P$. falciparum lipids and the presence of cross-reactive oligosaccharide epitopes in P. falciparum-infected red blood cells [18]. Plasmodium falciparum lacks genes for many glycosyl transferases involved in synthesising $\mathrm{N}$-glycans, glycophosphatidylinositol anchors and $\mathrm{O}$-glycans compared to other eukaryotes $[19,20]$. In vitro cultures of $P$. falciparum asexual blood stages did not incorporate galactose from UDP-galactose into glycolipids and glycoproteins when compared with parallel cultures of Trypanosoma brucei promastigotes [18]. BLASTp searches of the P. falciparum genome (GeneDB, Wellcome-Sanger Institute) with mammalian and other galactosyl transferases including a digalactosyl diglyceride synthase from Arabidopsis thaliana did not identify homologous proteins [18]. However, a Staphylococcus aureus galactosyl transferase with a function in synthesising cell wall components was significantly homologous to a $P$. falciparum $N$-acetylglucosaminyl phosphatidyl inositol synthase, an enzyme with a role in synthesising glycophosphatidylinositol membrane anchors [18], showing that BLASTp searches can reveal homologies in evolutionarily widely divergent organisms. These reports in conjunction with the present findings suggest that Plasmodium lacks the genetic ability to synthesise $\alpha$-gal-containing glycoconjugates. This is consistent with the strong immune selection likely against malaria parasites expressing $\alpha$-gal in catarrhines possessing natural antibodies to $\alpha$-gal [21]. On the other hand, minimal or no immune selection against the expression of $\alpha$-gal in vector mosquitoes is expected because their interaction with catarrhines only involves blood feeding.

The alternative of a mosquito origin for the $\alpha$-gal detected on P. berghei and P. falciparum sporozoites [11] is therefore a likely explanation, as also proposed elsewhere $[21,22]$. In migrating to salivary ducts from the haemocoele, Plasmodium sporozoites traverse a basement membrane and then move through salivary gland epithelial cells with many close intermolecular interactions occurring between the sporozoites and mosquito 
cells [23]. Mosquito-derived glycoconjugates can be transferred to the sporozoite membrane during these processes. This interpretation is consistent with the present finding of several putative GTs homologous to I. scapularis a1-3 GTs in An. gambiae and Ae. aegypti. It is also supported by demonstration of the transfer of labelled synthetic galactosyl glycoconjugates from Anopheles stephensi to P. berghei sporozoites in salivary glands [22], and the synthesis of $\alpha$-gal in cultured cells derived from another important arboviral vector Aedes albopictus [24]. The presence of multiple proteins in An. gambiae and Ae. aegypti homologous to the three $I$. scapularis enzymes with $\alpha 1-3$ GT activity also suggests that the numbers of $\alpha$ and $\beta$ GTs in mosquitoes may be increased as in I. scapularis, and this warrants further investigation.

The presence of $\alpha 1-3$ GT activity in ticks and mosquitos and the possible inoculation of $\alpha$-gal-containing glycoconjugates into humans during blood feeding by the two types of arthropod vectors raise important issues regarding hypersensitivity reactions to their bites: (i) In the case of AGS hypersensitivity induced by tick vectors, it is not clear how IgE antibodies to $\alpha$-gal are produced in the face of overwhelming concentrations of IgG and IgM antibodies to $\alpha$-gal that might be expected to bind and remove relevant tick glycoconjugates from the inoculation site and blood. To explain this, it has been proposed that components such as prostaglandin E2 introduced with tick saliva during biting induce a $\mathrm{T}_{\mathrm{H}} 2$ bias and immunoglobulin class switching that favour the formation of IgE antibodies to $\alpha$-gal [25]. It may also be relevant in this context that ticks are relatively rare human blood feeders. (ii) Mosquitos differ from ticks in that blood feeding is typically of a shorter duration, and bites are more frequent and may occur over many years in some locations. In comparison to tick bites, IgE and IgG antibodies may therefore have different temporal roles in the development of hypersensitivity to mosquito bites and an eventual desensitisation [1]. (iii) Little is presently known about the possible roles of $\alpha$-gal or other carbohydrate epitopes in salivary glycoconjugates in causing hypersensitivity to mosquito bites. This gap in knowledge needs to be addressed.

Allergen immunotherapy with natural and recombinant salivary gland proteins is being developed for treating both AGS [4] and hypersensitivity caused by mosquito bites [1]. The possible effects of $\alpha$-gal that can be present in the immunogens used for immunotherapy, however, requires careful consideration.

The four common human malaria parasite species, all of which had origins in African apes, have caused major genetic changes in humans associated with resistance to malaria [26]. It has been speculated that a selective advantage conferred by resistance to a pathogen [9], possibly an ancestral malaria parasite [7,27], led to the inactivation of $\alpha 1-3$ GTs in catarrhines. The present data raise the possibility that neutralising the infective stages of pathogens, particularly ancestral malaria parasites, carrying vector-derived $\alpha$-gal, and amelioration of hypersensitivity reactions may have been selective forces favouring the ability to make IgG and IgM antibodies to $\alpha$-gal through the inactivation of $\alpha 1-3$ GT in catarrhines. The use of a variety of immunogens possessing terminal $\alpha$-galactosyl residues to vaccinate against multiple human parasites (e.g. Plasmodium species, Leishmania species and Trypanosoma cruzi), human bacterial pathogens (e.g. Borrelia burgdorferi, Anaplasma phagocytophilum and Mycobacterium species) and veterinary pathogens (e.g. Aspergillus fumigatus, Newcastle disease virus) [28], as well as enveloped zoonotic viruses that infect humans (e.g. SARS-CoV-2) [29], has been proposed. The high concentration of natural antibodies to $\alpha$-gal in blood and hypersensitivity reactions are factors that have to be carefully considered in this respect.

Because of likely similar evolution among arthropods, the mosquito proteins identified here are good candidates for enzymes involved in synthesising the $\alpha$-gal that has been reported to be present in mosquito cells $[11,24]$. Experimental verification of the synthesis of $\alpha$-gal in mosquito salivary glands by the putative identified enzymes, and its transfer to Plasmodium sporozoites, is now needed to further understand primate evolution, hypersensitivity to mosquito bites and protection against malaria and other mosquito-borne pathogens.

\section{Abbreviations}

a-gal: Gala1-3Galß1-4GIcNAc-R; AGS: a-Gal syndrome; BLASTp: Basic Local Alignment Search Tool for proteins; Gal: Galactose; Gb: Globoside; GlcNAc: N-Acetyl glucosamine; GT: Galactosyl transferase; NCBI: National Center for Biotechnology Information; SARS-CoV-2: Severe acute respiratory syndrome coronavirus $2 ; T_{H} 2: T$ helper type 2 lymphocyte.

\section{Supplementary Information}

The online version contains supplementary material available at https://doi. org/10.1186/s13071-021-04801-7.

Additional file 1. Phylogenetic distance trees constructed with the NCBI BLASTp results.

\section{Acknowledgements}

Not applicable.

Authors' contributions

The author read and approved the final manuscript.

Funding

Not applicable. 


\section{Availability of data and materials}

All data generated or analysed during this study are included in this published article and its Additional file 1.

\section{Declarations}

Ethics approval and consent to participate

Not applicable.

\section{Consent for publication}

Not applicable.

\section{Competing interests}

The author declares no competing interests.

Received: 1 February 2021 Accepted: 24 May 2021

Published online: 05 June 2021

\section{References}

1. Peng Z, Simons FE. Mosquito allergy: immune mechanisms and recombinant salivary allergens. Int Arch Allergy Immunol. 2004;133:198-209.

2. Centers for Disease Control and Prevention, Atlanta, GA, USA:Tick-borne diseases. 2011. https://www.cdc.gov/niosh/topics/tick-borne/resources. html. Accessed 11 Jan 2021.

3. Narasimhan S, Kurokawa C, Diktas H, Strank NO, Černý J, Murfin K, et al. Ixodes scapularis saliva components that elicit responses associated with acquired tick-resistance. Ticks Tick Borne Dis. 2020;11:101369.

4. Platts-Mills TAE, Commins SP, Biedermann T, van Hage M, Levin M, Beck $L A$, et al. On the cause and consequences of IgE to galactose- $a-1,3-$ galactose: a report from the National Institute of Allergy and Infectious Diseases Workshop on understanding IgE-mediated mammalian meat allergy. J Allergy Clin Immunol. 2020;145:1061-71.

5. Hamadeh RM, Galili U, Zhou P, Griffiss JM. Anti-alpha-galactosyl immunoglobulin A (lgA), IgG, and IgM in human secretions. Clin Diagn Lab Immunol. 1995;2:125-31.

6. Macher BA, Galili U. The Gal alpha1,3 Gal beta1,4GlcNAc-R (alpha-Gal) epitope: a carbohydrate of unique evolution and clinical relevance. Biochim Biophys Acta. 2008;1780:75-88.

7. Ramasamy R, Rajakaruna R. Association of malaria with inactivation of alpha1,3-galactosyl transferase in catarrhines. Biochim Biophys Acta. 1997;1360:241-6

8. Montassier E, Al-Ghalith GA, Mathé C, Le Bastard Q, Douillard V, Garnier $A$, et al. Distribution of bacterial a1,3-galactosyltransferase genes in the human gut microbiome. Front Immunol. 2020:10:3000.

9. Galili U, Swanson K. Gene sequences suggest inactivation of alpha1,3-galactosyltransferase in catarrhines after the divergence of apes from monkeys. Proc Natl Acad Sci USA. 1991;88:7401-4.

10. Cabezas-Cruz A, Espinosa PJ, Alberdi P, Šimo L, Valdés JJ, Mateos-Hernández $L$, et al. Tick galactosyltransferases are involved in a-Gal synthesis and play a role during Anaplasma phagocytophilum infection and Ixodes scapularis tick vector development. Sci Rep. 2018:8:14224.

11. Yilmaz B, Portugal S, Tran TM, Gozzelino R, Ramos S, Gomes J, et al. Gut microbiota elicits a protective immune response against malaria transmission. Cell. 2014;159:1277-89.
12. Zhang Y Wang PG, Brew K. Specificity and mechanism of metal ion activation in UDP-galactose: $\beta$-galactoside- $a-1,3$ galactosyl transferase. J Biol Chem. 2001:276:11567-74.

13. Flores-Garcia Y, Nasir G, Hopp CS, Munoz C, Balaban AE, Zavala F, et al. Antibody-mediated protection against Plasmodium sporozoites begins at the dermal inoculation site. MBio. 2018;9:e02194-18.

14. Gueirard P, Tavares J, Thiberge S, Bernex F, Ishino T, Milon G, et al. Development of the malaria parasite in the skin of the mammalian host. Proc Natl Acad Sci USA. 2010;107:18640-5.

15. Ramasamy R, Reese RT. A role for carbohydrate moieties in the immune response to malaria. J Immunol. 1985;134:1952-5.

16. Ramasamy R, Reese RT. Terminal galactose residues and the antigenicity of Plasmodium falciparum glycoproteins. Mol Biochem Parasitol. 1986:19:91-101.

17. Ramasamy R. Studies on glycoproteins in the human malaria parasite Plasmodium falciparum-lectin binding properties and the possible carbohydrate-protein linkage. Immunol Cell Biol. 1987;65:147-52.

18. Ramasamy R, Field MC. Terminal galactosylation of glycoconjugates in Plasmodium falciparum asexual blood stages and Trypanosoma brucei bloodstream trypomastigotes. Exp Parasitol. 2012;130:314-20.

19. Samuelson J, Banerjee S, Magnelli P, Cui J, Kelleher DJ, Gilmore R, et al. The diversity of dolichol-linked precursors to Asn-linked glycans likely results from secondary loss of sets of glycosyltransferases. Proc Natl Acad Sci USA. 2005:102:1548-53.

20. Cova M, Rodrigues JA, Smith TK, Izquierdo L. Sugar activation and glycosylation in Plasmodium. Malar J. 2015;14:427.

21. Ramasamy R. Evaluation of the importance of antibodies to a-galactosyl epitopes in immunity to malaria. J Vector Borne Dis. 2015;52:337-8.

22. Kitowski A, Bernardes GJL. A sweet galactose transfer: metabolic oligosac charide engineering as a tool to study glycans in Plasmodium infection. ChemBioChem. 2020;21:2696-700.

23. Mueller AK, Kohlhepp F, Hammerschmidt C, Michel K. Invasion of mosquito salivary glands by malaria parasites: prerequisites and defense strategies. Int J Parasitol. 2010;40:1229-35.

24. Lei $Y, Y u$ H, Dong Y, Yang J, Ye W, Wang Y, et al. Characterization of $\mathrm{N}$-glycan structures on the surface of mature dengue 2 virus derived from insect cells. PLoS ONE. 2015;10:e0132122.

25. Cabezas-Cruz A, Mateos-Hernández L, Chmelař J, Villar M, de la Fuente J. Salivary prostaglandin E2: role in tick-induced allergy to red meat. Trends Parasitol. 2017;33:495-8.

26. Ramasamy R. Zoonotic malaria-global overview and research and policy needs. Front Public Health. 2014;2:123.

27. Ramasamy R. Is malaria linked to the absence of alpha-galactosyl epitopes in Old World primates? Immunol Today. 1994;15:140.

28. Hodžić A, Mateos-Hernández L, de la Fuente J, Cabezas-Cruz A. a-Galbased vaccines: advances, opportunities, and perspectives. Trends Parasitol. 2020;36:992-1001.

29. Galili U. Host synthesized carbohydrate antigens on viral glycoproteins as "achilles' heel" of viruses contributing to anti-viral immune protection. Int J Mol Sci. 2020;21:6702.

\section{Publisher's Note}

Springer Nature remains neutral with regard to jurisdictional claims in published maps and institutional affiliations. 